(2) Open Access Full Text Article

\title{
Investigation of the Active Ingredients and Mechanism of Polygonum cuspidatum in Asthma Based on Network Pharmacology and Experimental Verification
}

This article was published in the following Dove Press journal:

Drug Design, Development and Therapy

Junjie $B i^{1, *}$

Yuhua Lin $\mathbb{D}^{1, *}$

Yipeng Sun ${ }^{1, *}$

Mengzhe Zhang ${ }^{2, *}$

Qingge Chen'

Xiayi Miu'

Lingling Tang'

Jinjin Liu'

Linyun Zhu'

Zhenhua $\mathrm{Ni}^{3}$

Xiongbiao Wang'

'Department of Respiratory Medicine, Putuo Hospital, Shanghai University of Traditional Chinese Medicine, Shanghai, 200062, People's Republic of China;

${ }^{2}$ Department of Laboratory Medicine, Putuo Hospital, Shanghai University of Traditional Chinese Medicine, Shanghai, 200062, People's Republic of China; ${ }^{3}$ Central Laboratory, Putuo Hospital, Shanghai University of Traditional Chinese Medicine, Shanghai, 200062, People's Republic of China

*These authors contributed equally to this work

\begin{abstract}
Background: Polygonum cuspidatum is a Chinese medicine commonly used to treat phlegmheat asthma. However, its anti-asthmatic active ingredients and mechanism are still unknown. The aim of this study was to predict the active ingredients and pathways of Polygonum cuspidatum and to further explore the potential molecular mechanism in asthma by using network pharmacology. Methods: The active ingredients and their targets related to Polygonum cuspidatum were seeked out with the TCM systematic pharmacology analysis platform (TCMSP), and the ingredient-target network was constructed. The GeneCards, DrugBank and OMIM databases were used to collect and screen asthma targets, and then the drug-target-disease interaction network was constructed with Cytoscape software. A target protein-protein interaction (PPI) network was constructed using the STRING database to screen key targets. Finally, GO and KEGG analyses were used to identify biological processes and signaling pathways. The anti-asthmatic effects of Polygonum cuspidatum and its active ingredients were tested in vitro for regulating airway smooth muscle (ASM) cells proliferation and MUC5AC expression, two main symptoms of asthma, by using Real-time PCR, Western blotting, CCK-8 assays and annexin V-FITC staining.
\end{abstract}

Results: Twelve active ingredients in Polygonum cuspidatum and 479 related target proteins were screened in the relevant databases. Among these target proteins, 191 genes had been found to be differentially expressed in asthma. PPI network analysis and KEGG pathway enrichment analysis predicted that the Polygonum cuspidatum could regulate the AKT, MAPK and apoptosis signaling pathways. Consistently, further in vitro experiments demonstrated that Polygonum cuspidatum and resveratrol (one active ingredient of Polygonum cuspidatum) were shown to inhibit ASM cells proliferation and promoted apoptosis of ASM cells. Furthermore, Polygonum cuspidatum and resveratrol inhibited PDGF-induced AKT/ mTOR activation in ASM cells. In addition, Polygonum cuspidatum decreased $\mathrm{H}_{2} \mathrm{O}_{2}$ induced MUC5AC overexpression in airway epithelial NCI-H292 cells.

Conclusion: Polygonum cuspidatum could alleviate the symptoms of asthma including ASM cells proliferation and MUC5AC expression through the mechanisms predicted by network pharmacology, which provides a basis for further understanding of Polygonum cuspidatum in the treatment of asthma.

Keywords: Polygonum cuspidatum, asthma, ASM cells, MUC5AC, network pharmacology

\section{Introduction}

Bronchial asthma (asthma) is a common chronic respiratory airway inflammatory disease. Its clinical manifestations are airway inflammation, airway hyperresponsiveness
Wang

Email zhenhuani@yeah.net;

xiongbiao6@yahoo.com
Drug Design, Development and Therapy 2021:15 1075-1089

1075 
and airway remodeling. ${ }^{1,2}$ Its incidence rate is increasing, and it seriously affects human health and quality of life because of the recurrence and the high cost of medical treatment. Currently, some patients with refractory asthma cannot be effectively controlled by corticosteroid treatment. Therefore, it is necessary to develop alternative treatments for asthma. Clinical findings show that the use of traditional Chinese medicine (TCM), a type of alternative medicine, has achieved good clinical effects. ${ }^{3-5}$ As a supplementary medicine, TCM has been increasingly applied in the treatment of asthma, and the treatment is effective, with few side effects. ${ }^{6}$

The herbal Polygonum cuspidatum (huzhang) has been recorded in many medical books. It has the effects of clearing away heat and phlegm, relieving cough, relieving asthma, dispersing blood stasis and relieving pain. ${ }^{7}$ Several studies indicate that Polygonum cuspidatum has the effects of inhibiting liver fat accumulation, preventing diabetes, treating gout arthritis, and treating ulcerative colitis and cancer. ${ }^{8-12}$ Polygonum cuspidatum is a herbal medicine commonly used to treat phlegm-heat asthma. Our previous study showed that the Chinese herbal Qixian decoction (containing Polygonum cuspidatum) exhibited therapeutic effects for asthma treatment. ${ }^{13}$ However, the mechanism of the treatment in asthma by Polygonum cuspidatum has not been elucidated. Therefore, this study aims to assess the effects of Polygonum cuspidatum on asthma and determine its biological targets and molecular mechanism to provide a scientific reference for further research.

As we know, the complex composition and unclear mechanism of herbs limit its further application. Numerous experiments are needed to screen the active ingredients and potential targets usually. Network pharmacology provides a new method and new concept for natural herbs to study the mechanisms of certain ingredient by computational pharmacology from existed research results. Network pharmacology assumes that the pharmacological effect of drugs is generated by inducing a change in the interaction network of biological macromolecules in organisms, in addition to specific drug target interactions. This is compatible with the overall concept of traditional Chinese medicine and the multiple targets of traditional Chinese medicine treatment, which provides a great opportunity for the modernization of traditional Chinese medicine.

Here, we performed a network pharmacologic analysis of Polygonum cuspidatum in asthma treatment. Then, we validated the results obtained from the network pharmacology prediction and explored the molecular mechanism of
Polygonum cuspidatum and its active ingredients in preventing asthma symptoms including airway smooth muscle (ASM) cells proliferation and MUC5AC overexpression. This is the first study to investigate the underlying mechanism of Polygonum cuspidatum for the treatment of asthma by network pharmacology methods and experimental verification.

\section{Materials and Methods}

\section{Selection of the Active Ingredients from} Polygonum cuspidatum and Construction of the "Ingredient-Target" Network

TCMSP (http://lsp.nwu.edu.cn/tcmsp.php) was used for a search of Polygonum cuspidatum ingredients according to the following screening conditions: oral bioavailability (OB) $\geq 30 \%$ and drug-like (DL) $\geq 0.18{ }^{14}$ the results were then combined with the corresponding literature search results, ${ }^{15,16}$ and the active ingredients of Polygonum cuspidatum were screened. Then, TCMSP was used to screen the predicted target proteins of the active ingredients, and the UniProt network platform (https://www.uniport.org/) was used to integrate the targets protein, which were imported into Cytoscape 3.7.2 software to construct the "ingredient-target" network.

\section{Screening of Asthma Targets and Construction of a "Disease-Target" Network}

The DrugBank (https://www.drugbank.ca/), Genecards database (https://www.genecards.org/) and OMIM database (https://www.omim.org/) were used to search for asthma-related targets by using "asthma" as the key word. The target proteins were integrated through UniProt, and the results were imported into Cytoscape 3.7.2 software to build the "disease-target" network.

\section{Construction of the "Drug-Target- Disease" Network Involved in the} Treatment of Asthma with Polygonum cuspidatum

To clarify the interaction between the active ingredient targets of Polygonum cuspidatum and asthma targets, the identified "ingredient targets" and "disease targets" were mapped with Venny 2.1. Then, the intersection of the two groups of targets was used to determine the common targets of the active ingredient of Polygonum cuspidatum and disease, which was then imported into Cytoscape 3.7.2 
software to generate the network, and the "drug-targetdisease" visualization was constructed.

\section{Construction of the PPI Network and Core Target Selection}

The interactions between proteins are the basis of human life activities and are also important in exploring the molecular mechanisms of the body. To clarify the interaction between drugs and disease intersection targets, we used the STRING platform (https://string-db.org/) to screen the interactions between the targets, build a PPI network, and study disease classification from a systematic perspective to identify the mechanism and to discover new drug targets. Finally, the core targets were screened according to the degree parameter. ${ }^{17}$ Proteins with high values were considered to occupy the core positions in the PPI network and were more likely to serve as key core targets.

\section{KEGG Pathways of Potential Targets of Polygonum cuspidatum in the Treatment of Asthma}

$\mathrm{R}$ software version 3.6.2 and the Bioconductor software package were used to preprocess the intersection target proteins of the "drug-target-disease" network, and the Bioconductor software packages "dose", "clusterprofiler" and "pathview" were installed to analyze the KEGG pathways, determine the significance $(\mathrm{P}<0.05)$, and draw the bubble chart. In the bubble chart, the p.adjust value indicates the significance, and a larger circle indicates a greater number of enrichment targets and the greater importance of a signaling pathway.

\section{Reagents}

The reagents used in the study were as follows: DMEM (Thermo Fisher scientific, Waltham, MA, USA), fetal bovine serum (Thermo Fisher Scientific, USA), Cell Counting Kit-8 and Annexin-V apoptosis detection kit (Dojindo, Kumamoto, Japan), LY294002 (Selleck, Houston, USA) and rapamycin (Selleck, USA), BCA protein assay kit, and Cell lysis reagent (Beyotime Biotechnology, Jiangsu, China), antibodies against p-AKT, p-mTOR (Cell Signaling Technology, Danvers, USA), antibodies against $\beta$-actin (Abcam, Cambridge, USA), HRPlinked antibody (Cell Signaling Technology, USA), Recombinant Rat PDGF-BB (PeproTech, USA), resveratrol (Sigma, St. Louis, MO, USA), quercetin (Selleck, USA), polydatin (Absin, Shanghai, China), Polygonum cuspidatum (Lys Pharmaceutical Co., Ltd, Shanghai, China).

\section{Preparation and Characterization of Polygonum cuspidatum Extraction (PCE)}

Four liters of water were added to $100 \mathrm{~g}$ of Polygonum cuspidatum. After maceration for $1 \mathrm{~h}$, the herbs were boiled and concentrated to $0.8 \mathrm{~L}$. The concentrated decoction was then filtrated to remove insoluble sediment and used for in vitro study. The concentrations of resveratrol in the PCE were determined by HPLC method. Thermo U3000 HPLC system and Waters XTerra C18 column were used. With water as mobile phase A and acetonitrile as mobile phase B, elution gradients were as follows: 0-20 $\min (15 \%-32.5 \%$ B), $20-21 \min (32.5 \%-15 \%$ B), $25 \min (15 \%$ B), and column temperature was set at $35^{\circ} \mathrm{C}$. The flow rate was $1.0 \mathrm{~mL} / \mathrm{min}$ and the detection wavelength was set at $290 \mathrm{~nm}$.

\section{Isolation, Culture and Identification of Rat ASM Cells}

The isolation of ASM cells was performed according to a previously reported method with some modifications. ${ }^{18}$ All animal experiments were performed in accordance with the NIH Guide for the Care and Use of Laboratory Animals and the procedures were subject to approval by the Institutional Animal Care and Use Committee, Putuo Hospital, Shanghai University of Traditional Chinese Medicine. Sprague-Dawley (SD) rats were anaesthetized with pentobarbital sodium, and the trachea was isolated aseptically and washed with normal saline (containing $100 \mathrm{u} / \mathrm{mL}$ penicillin and $100 \mathrm{u} / \mathrm{mL}$ streptomycin). The trachea was transferred to DMEM with $20 \%$ FBS, and the outer membrane was peeled away carefully with aseptic cotton. The smooth muscle was isolated, cut into sections of 1-2 $\mathrm{mm}$ in cubic size, and cultured upside down for $4 \mathrm{~h}$ in culture flasks with DMEM with $20 \% \mathrm{FBS}$ in an incubator at $37^{\circ} \mathrm{C}$ in $5 \% \mathrm{CO}_{2}$. The culture medium was changed every 3 days. The ASM cells were purified by differential attachment to remove fibroblasts and identified by immunofluorescence staining using an anti- $\alpha$-SMA antibody. ASM cells were maintained in DMEM (Thermo Fisher Scientific, Waltham, MA, United States) supplemented with $10 \%$ foetal bovine serum, penicillin, and streptomycin. Cells were maintained in an incubator at $37^{\circ} \mathrm{C}$ with $5 \% \mathrm{CO}_{2}$. 


\section{Cell Viability Assays}

Cell viability was monitored by using a Cell Counting Kit-8 (CCK-8, Dojindo, Japan) according to the manufacturer's instructions. Briefly, cells were seeded at a density of $1 \times 10^{4}$ cells/well in 96-well plates, and incubated in DMEM supplemented with FBS overnight. Cells were treated with various concentrations of resveratrol or quercetin, or LY294002 for the indicated time in DMEM with 2\% FBS and appropriate controls were treated with DMSO at the same concentrations. CCK-8 solution $(100 \mu \mathrm{L})$ was added to each well for $2 \mathrm{~h}$ and optical density was measured at $450 \mathrm{~nm}$ to estimate viable cells.

\section{Cell Apoptosis Assays}

The Annexin V-FITC Apoptosis Detection Kit (Dojindo, Japan) was utilized according to the manufacturer's protocol. In brief, floating and harvested cells were mixed, washed twice with PBS and resuspended in binding buffer. Annexin V-FITC $(5 \mu \mathrm{L})$ were added to $100 \mu \mathrm{L}$ of the cell suspension containing $10^{5}$ cells. The cell suspension was mixed and then incubated for $15 \mathrm{~min}$ at room temperature in the dark. Subsequently, $400 \mu \mathrm{L}$ of binding buffer were added and cells were analyzed by flow cytometry using Calibur.

\section{Real-Time Quantitative PCR}

The total RNA was isolated using Trizol reagent (TaKaRa, Dalian, China) and first-strand cDNAs were prepared using the First-Strand Synthesis Kit (TaKaRa, Dalian, China). Realtime PCR was performed using Master Mixer (TaKaRa, Dalian, China) and specific forward and reverse primers (Table 1). The relative expression levels of the target gene were normalized against $\beta$-actin analyzed via the $2^{-\Delta \Delta \mathrm{Ct}}$ method $\Delta \mathrm{Ct}=\left(\mathrm{Ct}_{\text {target }}-\mathrm{Ct}_{\text {actin }}\right)$ and $\Delta \mathrm{Ct}=\left(\mathrm{Ct}_{\text {target }}-\mathrm{Ct}_{\text {actin }}\right)$.

\section{Western Blots}

Western blot analysis was carried out according to published methods. ${ }^{19}$ Cells were washed once PBS and dissolved in cell lysis reagent (Cell Signaling Technology, USA). Total protein was separated with $10 \%$ SDS-PAGE, followed by transfer to a PVDF membrane. The PVDF membrane was blocked with $5 \%$ BSA, washed three times with TBST, and

Table I Sequences of Primers

\begin{tabular}{|l|c|}
\hline Primers & Sequences \\
\hline MUC5AC-F & 5- GCTTCCTGCTCCGAGATGT-3 \\
MUC5AC-R & 5- AAGACGCAGCCCTCATAGAA-3 \\
$\beta$-actin-F & 5-CCAACCGCGAGAAGATGA-3 \\
$\beta$-actin-R & 5-CCAGAGGCGTACAGGGATAG-3 \\
\hline
\end{tabular}

then incubated with the indicated antibodies separately overnight at $4^{\circ} \mathrm{C}$. The membrane was then washed with TBST three times followed by incubation with anti-rabbit IgG horseradish peroxidase secondary antibody (Cell Signaling Technology, USA) for $2 \mathrm{~h}$ at room temperature. Finally, immunoreactive bands were visualized with ECL reagent. Comparative protein expression levels were quantified by using Image $\mathbf{J}$ software and normalized to $\beta$-actin.

\section{Statistical Analysis}

The SPSS version 22.0 software and GraphPad Prism 8.0 were used for data analysis. The results were expressed in the form of mean \pm standard deviation (SD). Student's $t$-test, one-way ANOVA or two-way ANOVA methods were used. Statistically significant differences were defined as $p<0.05$.

\section{Results}

\section{Active Ingredients of Polygonum cuspidatum and Construction of a "Drug- Target-Disease" Network}

The flowchart of the whole study design is illustrated in Figure 1. According to the criteria of $\mathrm{OB} \geq 30 \%$ and $\mathrm{DL} \geq$ 0.18 in the TCMSP database, 10 key active ingredients and 324 related target proteins of Polygonum cuspidatum were obtained. Another 2 active ingredients, namely, resveratrol and polydatin, were also included for further analysis according to previous studies. ${ }^{15,20}$ Finally, 12 active ingredients of Polygonum cuspidatum were selected for further protein target prediction (Table 2). The main active ingredients were quercetin, resveratrol, luteolin, beta-sitosterol and physiovenene. A total of 5208 asthma-related target proteins were screened from the DrugBank, GeneCards database and OMIM database. A total of 191 asthma-related target proteins were identified from the intersection of drugs and diseases (Figure 2A, Table S1). The abovementioned active ingredients, drugtargeted proteins and drug-disease-targeted proteins were imported into Cytoscape 3.7.2 software for network visualization, and the "drug-target-disease" network was built (Figure 2B). From the Figure 2B, we could know resveratrol is the first active ingredients of the enrichment target genes, which indicated the predominant and primary bioactive substances mediated the anti-asthmatic effects of Polygonum cuspidatum.

\section{PPI Network Construction and Core Target Selection}

The 191 intersection targets were inputted into STRING to analyze the correlation between them and to build a PPI 


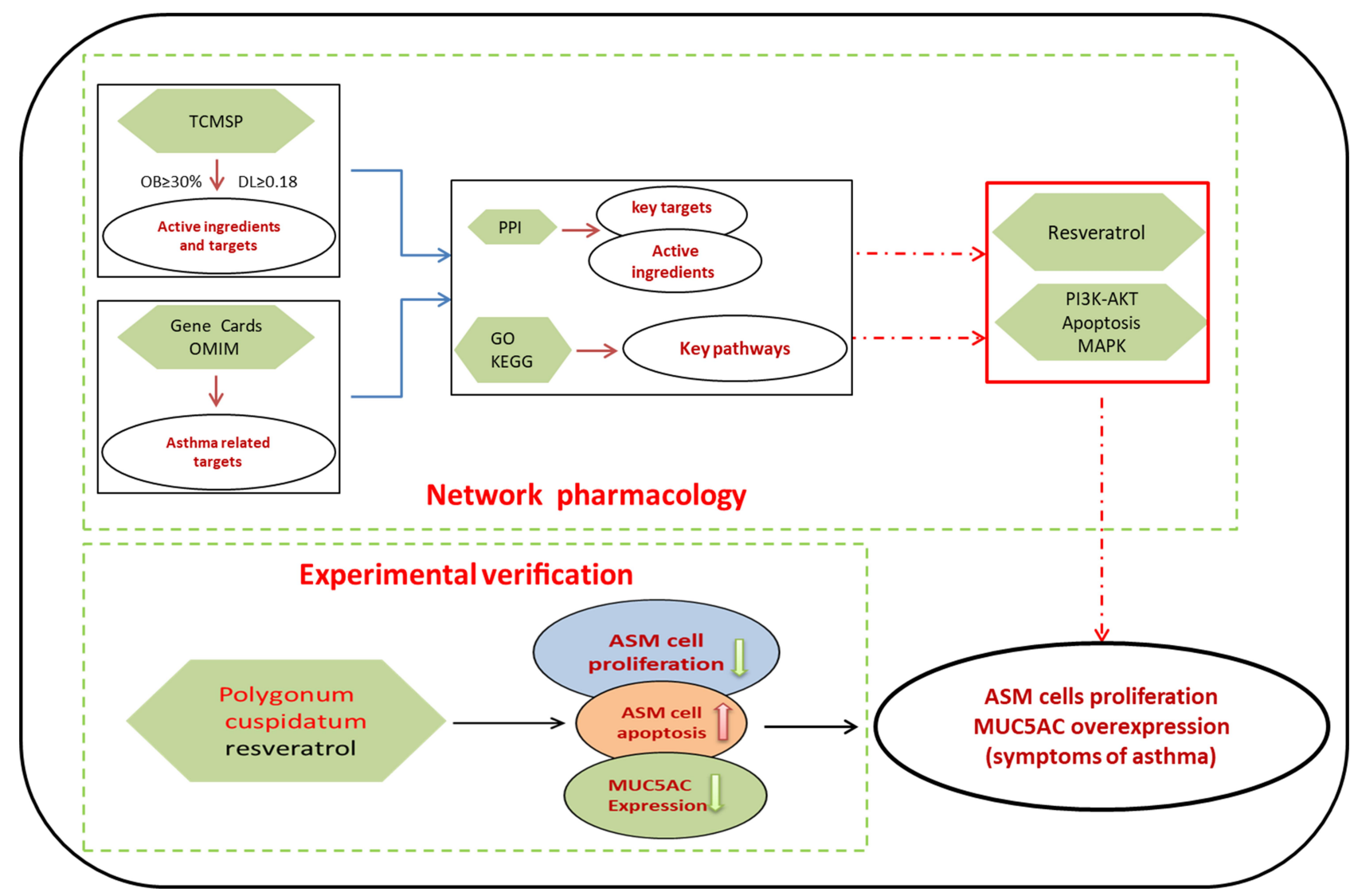

Figure I Entire study design based on network pharmacology and experimental verification.

visualization network (Figure 3A, Table S1). According to the distribution of the network degree, the core target proteins in the PPI network were obtained, and the first 30 core targets were screened (Figure 3B). The main core targets included AKT1, IL6, VEGFA, mapk3, CASP3, Jun, mapk8, EGFR, Myc, and STAT3. Therefore, these targets may be the key therapeutic targets of Polygonum cuspidatum in the treatment of asthma. Among the main core targets, AKT was the highest one.
Enrichment Analysis of the KEGG Pathways Involved in the Treatment of Asthma with Polygonum cuspidatum

After the enrichment analysis of the KEGG pathways, 165 signaling pathways were obtained. According to the number of enriched genes and the p.adjust value, the first 20 pathways were screened (Figure 4). Among these

Table 2 Main Active Ingredients and Target Numbers of Polygonum cuspidatum

\begin{tabular}{|c|c|c|c|c|}
\hline Mol ID & Molecule Name & OB (\%) & DL & Target \\
\hline MOL0I328I & 6,8-Dihydroxy-7-methoxyxanthone & 35.83 & 0.21 & 13 \\
\hline MOLOI3287 & Physovenine & 106.21 & 0.19 & 38 \\
\hline MOL0I3288 & Picralinal & 58.01 & 0.75 & 4 \\
\hline MOL002259 & Physciondiglucoside & 41.65 & 0.63 & I \\
\hline MOL002268 & Rhein & 47.07 & 0.28 & 7 \\
\hline MOL002280 & Torachrysone-8-O-beta-D-(6'-oxayl)-glucoside & 43.02 & 0.74 & I \\
\hline MOL000358 & Beta-sitosterol & 36.91 & 0.75 & 38 \\
\hline MOL000492 & $(+)$-catechin & 54.83 & 0.24 & 11 \\
\hline MOL000006 & Luteolin & 36.16 & 0.25 & 57 \\
\hline MOL000098 & Quercetin & 46.43 & 0.28 & 154 \\
\hline MOL0I3289 & Polydatin & 21.44 & 0.5 & 4 \\
\hline MOLOI 2744 & Reveratrol & 19.07 & 0.11 & $|5|$ \\
\hline
\end{tabular}



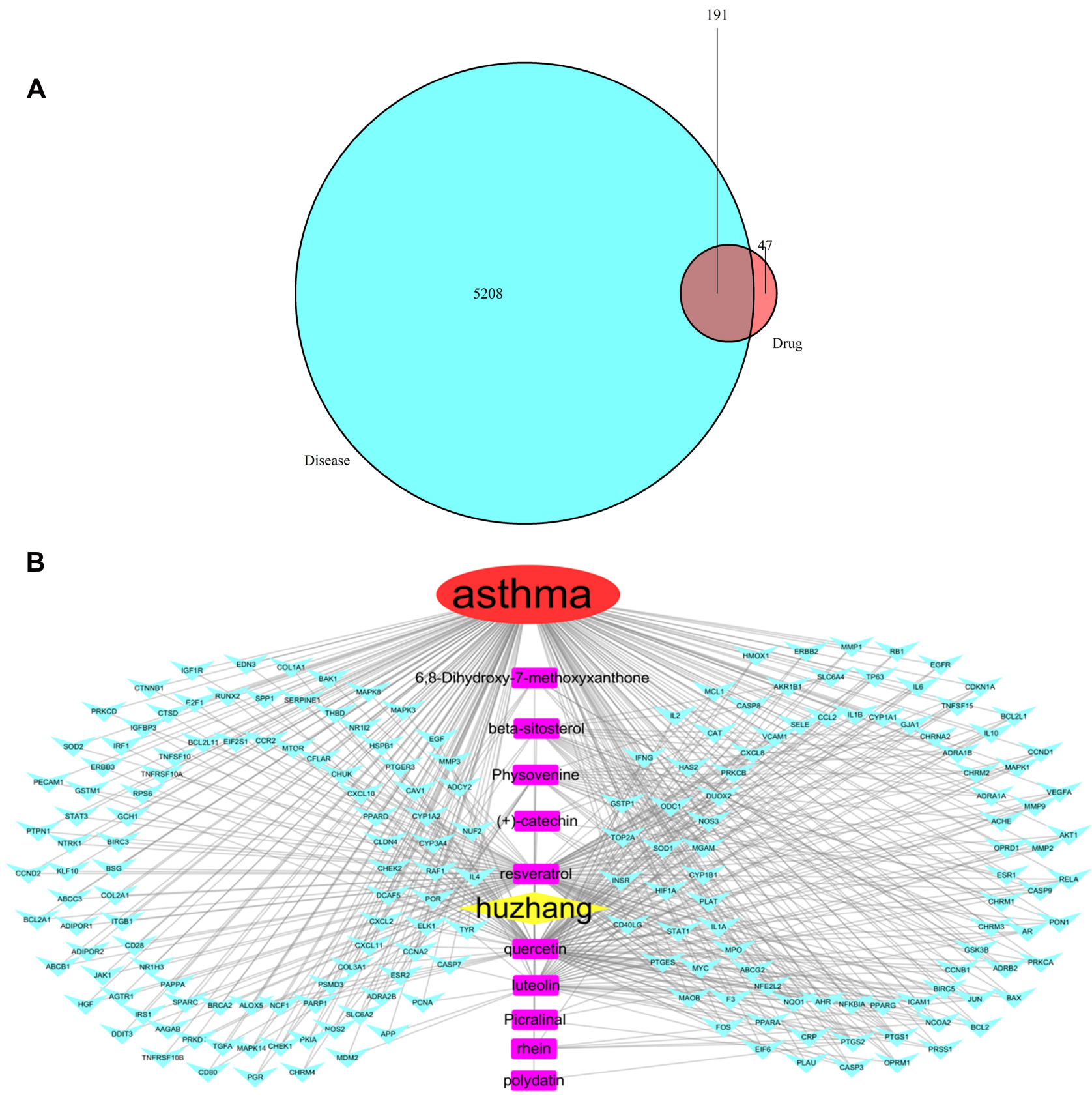

Figure 2 Disease and active ingredient intersection targets. (A) Intersection target Venn diagram. (B) "Drug-target-disease" network.

pathways, the AKT pathway had the highest protein enrichment, and the apoptosis pathway was the most significant, with the largest p.adjust value. This showed that Polygonum cuspidatum was involved in a multitarget and multichannel network. Among the involved proteins, the highest number involved was the PI3K-AKT signaling pathway, which was enriched in 42 target proteins (Table $\underline{\mathrm{S}}$ ). This shows that the main signaling pathway of Polygonum cuspidatum involved in the treatment of asthma may be the AKT signaling pathway.

\section{Polygonum cuspidatum Extraction Inhibits PDGF-Induced Proliferation of ASM Cells and AKT/mTOR Activation}

According to enrichment results of the KEGG signaling pathway analysis, we found that AKT signaling pathway played a key role in the effects of Polygonum cuspidatum. It had been reported AKT activation played an important role in the pathogenesis of asthma, such as by promoting ASM cell proliferation. ${ }^{21}$ We then verified whether Polygonum cuspidatum showed 
A

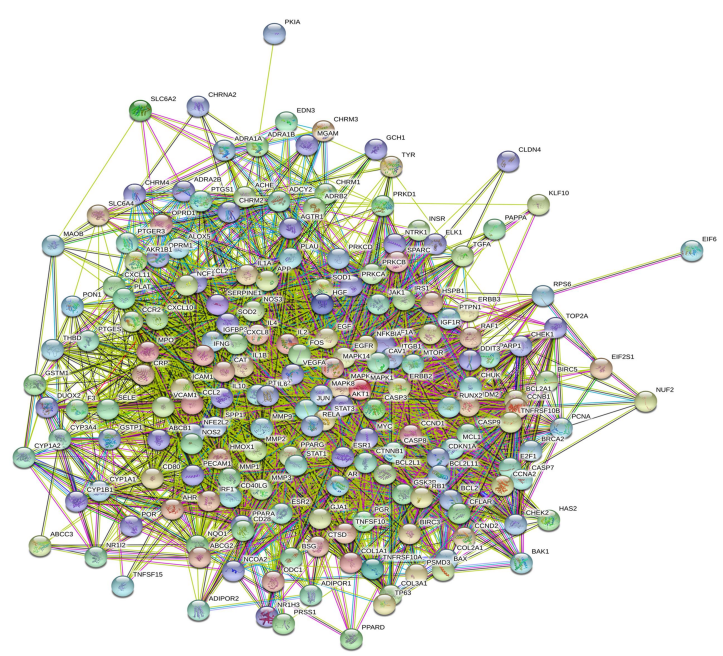

B

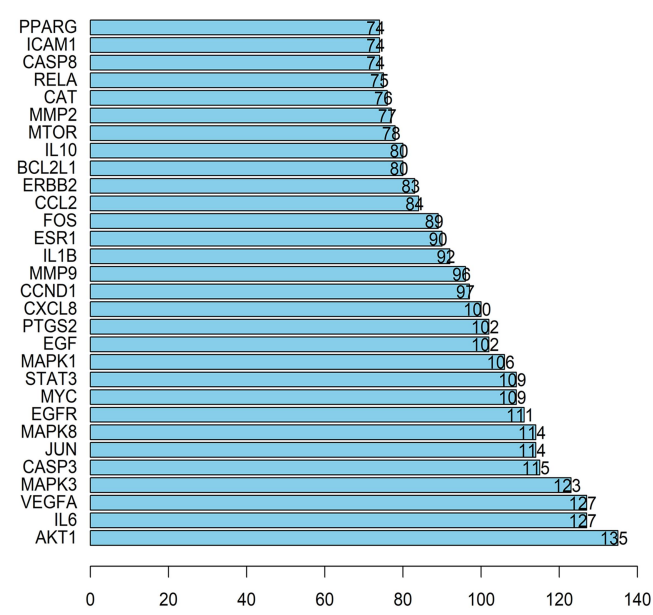

Figure 3 The key therapeutic targets of Polygonum cuspidatum in the treatment of asthma. (A) PPI visualization network. (B) Thirty core target selections. High value and high betweenness proteins are considered to occupy the core positions in the PPI network and are more likely to serve as key core targets.

an inhibitory effect on ASM proliferation. We used CCK-8 assays to analyse the effect of Polygonum cuspidatum extraction on the proliferation of ASM cells induced by platelet-derived growth factor (PDGF)-BB (a well-known ASM mitogen that promotes ASM proliferation). As showed in Figure 5, PDGF treatment promoted the proliferation of ASM cells, and Polygonum cuspidatum extraction significantly inhibited the proliferation of ASM cells in a dosedependent manner (Figure 5A). According to the network pharmacology analysis, the apoptosis pathway was found to be another significant pathway involved in the treatment of asthma with Polygonum cuspidatum (Figure 4 Table 2). Therefore, we examined cell apoptosis changes after Polygonum cuspidatum extraction treatment. We found that the apoptosis rate of ASM cells was markedly increased after Polygonum cuspidatum extraction treatment (Figure 5B and C). These results showed that Polygonum cuspidatum extraction could inhibit the proliferation of ASM cells induced by PDGF and induced apoptosis of ASM cells.

\section{Polygonum cuspidatum Extraction Inhibits PDGF-Induced AKT/mTOR Activation in ASM Cells}

We found that PDGF-BB significantly increased the phosphorylation levels of AKT and mTOR after $1 \mathrm{~h}$ and 5 h compared with those in the control group (Figure 6AC). Subsequently, we treated ASM cells with an AKT inhibitor (LY294002, $10 \mu \mathrm{M}$ ) and an mTOR inhibitor (rapamycin, $500 \mathrm{nM}$ ) for $5 \mathrm{~h}$ and found that inhibition of the AKT and mTOR signaling pathways suppressed the PDGF-induced proliferation of ASM cells (Figure 6D). Then, we treated ASM cells with PDGF plus Polygonum cuspidatum extraction $(0.25 \mathrm{mg} / \mathrm{mL}, 0.5 \mathrm{mg} / \mathrm{mL}, 1 \mathrm{mg} /$ $\mathrm{mL}, 2 \mathrm{mg} / \mathrm{mL}$ ), and found that Polygonum cuspidatum extraction could reduce the activation of AKT and mTOR induced by PDGF (Figure 7). These results indicated that the effects of Polygonum cuspidatum on ASM cell proliferation are associated with the AKT/mTOR signaling pathway.

\section{Polygonum cuspidatum Extraction Inhibits $\mathrm{H}_{2} \mathrm{O}_{2}$-Induced MUC5AC Overexpression in Airway Epithelial NCl-H292 Cells}

Based on enrichment results of the KEGG signaling pathway analysis, MAPK signaling pathway was another important target pathway of Polygonum cuspidatum. Several studies reported that asthma associated risk factors could cause pathologic activation of MAPK pathway leading to aggravation of symptoms of asthma such as MUC5AC overexpression. ${ }^{22,23}$ We then investigated the role of Polygonum cuspidatum on MUC5AC expression in airway epithelial NCI-H292 cells. We treated NCI$\mathrm{H} 292$ cells with $\mathrm{H}_{2} \mathrm{O}_{2}$ to induce MUCAC expression and activation of MAPK signaling pathway as previously described by Jang et $\mathrm{al}^{23}$ and found that upregulation of MUC5AC mRNA expression levels were reduced after Polygonum cuspidatum extraction treatment (Figure 8). These results suggested that Polygonum cuspidatum effectively inhibited $\mathrm{H}_{2} \mathrm{O}_{2}$-induced MUC5AC in NCIH292 cells. 
A

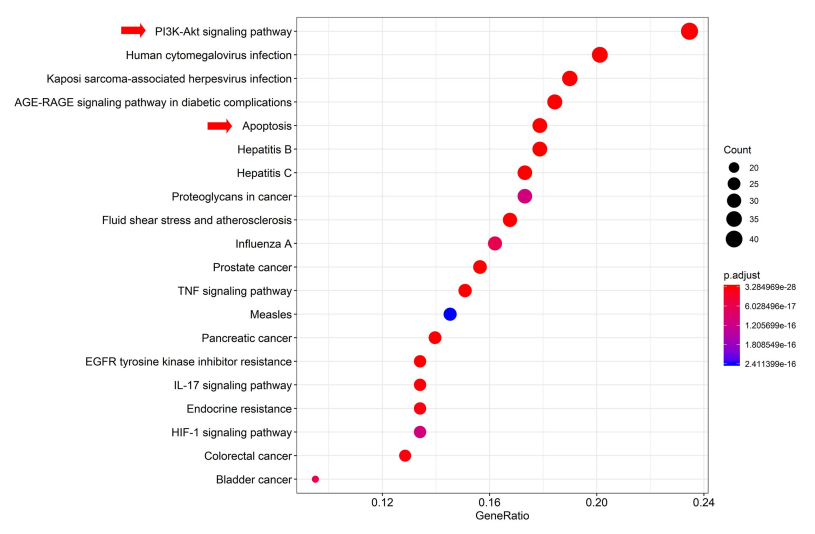

B

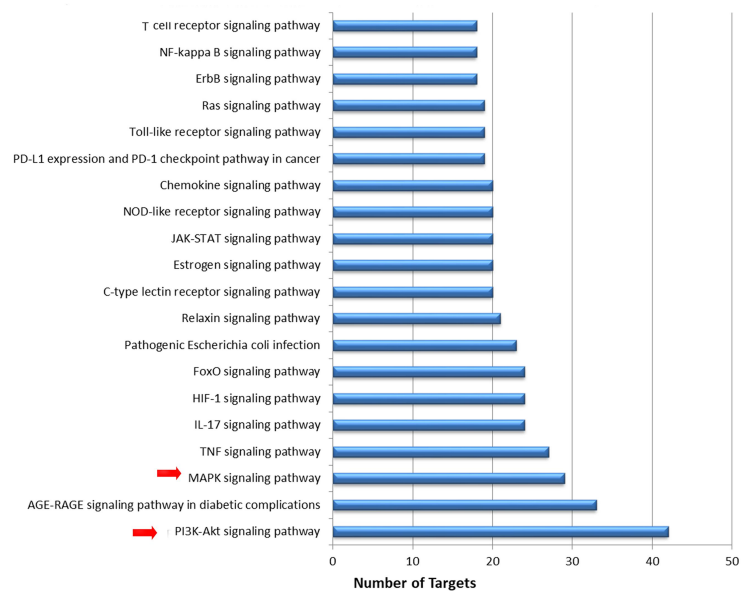

Figure 4 Enrichment analysis of KEGG pathways. (A) According to the p.adjust value of targets, the first 20 signaling pathways were involved in the treatment of asthma with Polygonum cuspidatum. In the bubble chart, the p.adjust value indicates significance, and the larger circles indicate a greater number of enrichment targets and the redder circles indicate greater significant targets. (B) According to the number of targets, the first 20 signaling pathways were involved.

\section{Resveratrol Inhibit PDGF-Induced Proliferation and AKT/mTOR Activation in ASM Cells}

Through the analysis of network pharmacology and related literature, resveratrol was found to have the most enrichment targets in polygonum cuspidatum. We then investigated whether the equivalent doses of resveratrol in Polygonum cuspidatum could have above biological effects. The concentrations of resveratrol in the Polygonum cuspidatum extraction were determined by HPLC. Four doses $(0.25 \mathrm{mg} / \mathrm{mL}, 0.5 \mathrm{mg} / \mathrm{mL}, 1 \mathrm{mg} / \mathrm{mL}$, $2 \mathrm{mg} / \mathrm{mL}$ ) of Polygonum cuspidatum were used for in vitro study, and the corresponding doses of resveratrol were $6.25 \mu \mathrm{M}, 12.5 \mu \mathrm{M}, 25 \mu \mathrm{M}$ and $50 \mu \mathrm{M}$. As showed in Figure 9, resveratrol significantly inhibited PDGF induced proliferation of ASM cells in a dose-dependent manner and promoted cell apoptosis at a high dose. Furthermore, the activation of AKT and mTOR induced by PDGF was markedly decreased after resveratrol treatment (Figure 10). These results showed that resveratrol was one of the important active ingredients in Polygonum cuspidatum to inhibit ASM cells proliferation.

\section{Discussion}

Polygonum cuspidatum has been applied to treat phlegmheat asthma in China. However, to date, few studies have been conducted the anti-asthmatic active ingredients and mechanism of Polygonum cuspidatum. In this study, we built a "Polygonum cuspidatum-targets-asthma" network.
Based on network pharmacology analysis, we identified the potential mechanisms of the anti-asthmatic effects of Polygonum cuspidatum and explored the possible active ingredients involved in preventing asthma. Moreover, our in vitro study demonstrated that Polygonum cuspidatum and its active ingredients resveratrol could alleviate asthma symptoms including inhibiting ASM cell proliferation and MUC5AC overexpression, indicating a promising anti-asthmatic mechanism and target of Polygonum cuspidatum for asthma treatment.

In this study, 12 active ingredients of Polygonum cuspidatum were selected for further analysis. Through the analysis of network pharmacology and related literature, resveratrol were found to have the most enrichment targets in Polygonum cuspidatum (Table 1, Figure 2B). Resveratrol was originally extracted from the roots of Polygonum cuspidatum and is also found in red wine, grapes, and peanuts. ${ }^{24}$ Moreover, resveratrol is the most studied monomer of Polygonum cuspidatum and is one of the major ingredients in Polygonum cuspidatum extract. ${ }^{12}$ Pan et al reported that 7 ingredients were identified from the crude extract of Polygonum cuspidatum using the UPLC-MS/MS method, including resveratrol. ${ }^{25}$ Several studies have suggested that resveratrol can help control the symptoms of asthma and improved the effectiveness of corticosteroid drug treatment in an asthma model. For example, the anti-inflammatory effects of resveratrol in asthma were associated with the inhibition of the production of inflammatory cytokines such as IL-4, IL-5 and IL13, the inhibition of TLR4/NFKB and the regulation of 
A

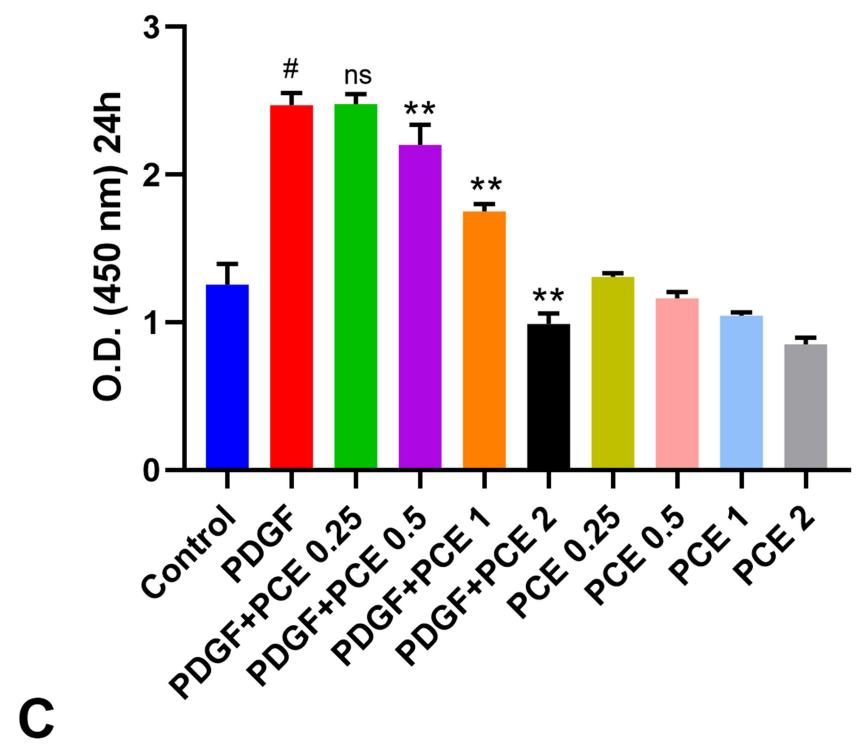

B

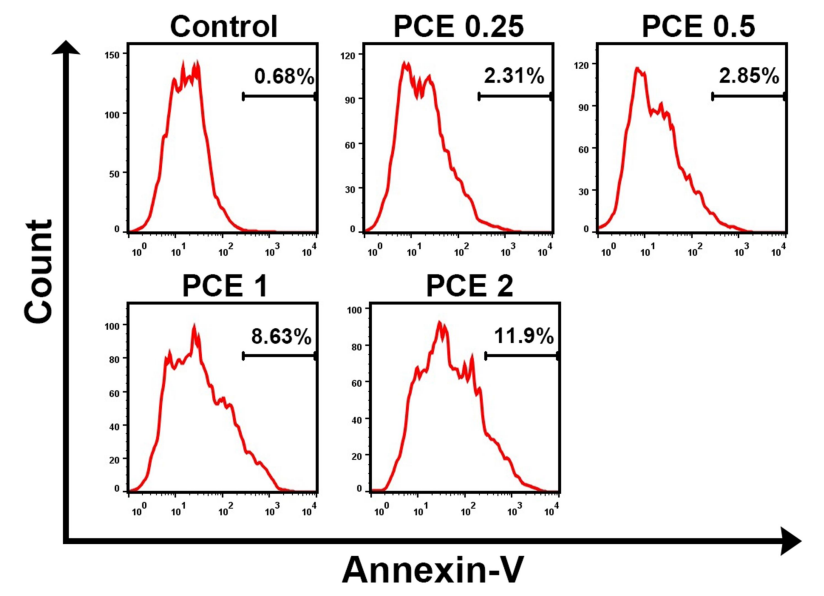

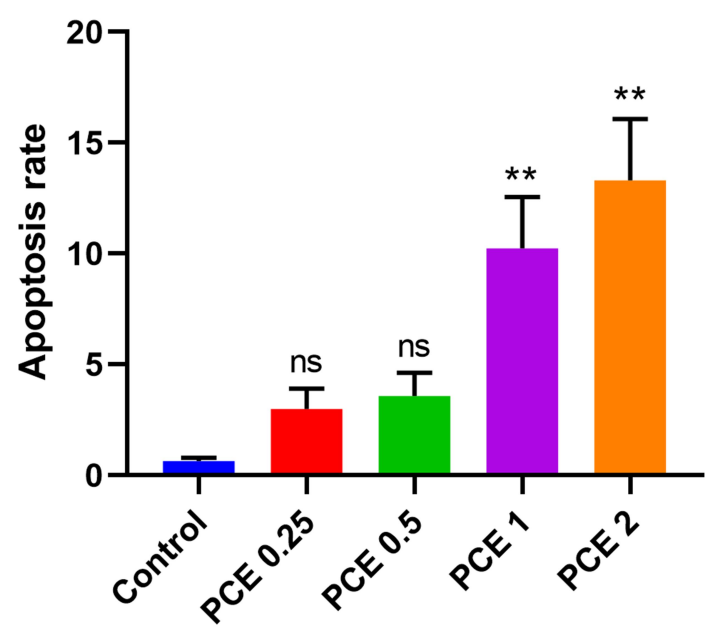

Figure 5 The effect of Polygonum cuspidatum extraction on ASM cells proliferation and apoptosis. (A) ASM cells were treated with PDGF (I0 ng/mL) and Polygonum cuspidatum extraction $(0.25 \mathrm{mg} / \mathrm{mL}, 0.5 \mathrm{mg} / \mathrm{mL}, 1 \mathrm{mg} / \mathrm{mL}$ and $2 \mathrm{mg} / \mathrm{mL})$ at different concentrations alone or in combination for $24 \mathrm{~h}$. Cell proliferation was detected by CCK8. The data were expressed as mean $\pm \mathrm{SD}(\mathrm{n}=6)$ of each group. (B, C) ASM cells were treated with Polygonum cuspidatum extraction $(0.25 \mathrm{mg} / \mathrm{mL}, 0.5 \mathrm{mg} / \mathrm{mL}, \mathrm{I} \mathrm{mg} / \mathrm{mL}$ and $2 \mathrm{mg} / \mathrm{mL}$ ) for $24 \mathrm{~h}$. The apoptosis of ASM cells were detected by Annexin-V staining. The data were expressed as mean \pm SD ( $n=3$ ) of each group. ${ }^{\text {ns }}$ P $>0.05$ vs PDGF group or control group and $* * \mathrm{P}<0.01$ vs $\mathrm{PDGF}$ group or control group, $\# \mathrm{P}<0.0$ Ivs control group.

miRNA-34a. ${ }^{16,26-29}$ These results were consistent with some previous reports that Polygonum cuspidatum has anti-inflammatory activities. ${ }^{30,31}$ In this study, we demonstrated that Polygonum cuspidatum and the equivalent doses of resveratrol exerted good anti-proliferation effects on ASM cells and promoted apoptosis. In asthmatic patients, ASM cells show excessive growth and insufficient apoptosis. The imbalance in ASM cells proliferation and apoptosis is closely linked to airway remodelling in asthma. Therefore, based on our results, it is likely that resveratrol is one of the important active ingredients of Polygonum cuspidatum to inhibit ASM cells proliferation.
Meanwhile our study demonstrated that Polygonum cuspidatum extraction at a dose of $0.5 \mathrm{mg} / \mathrm{mL}$ inhibited the proliferation of ASM cells induced by PDGF but the equivalent dose of resveratrol $(12.5 \mu \mathrm{M})$ did not. This suggested that other ingredients in Polygonum cuspidatum might participate in the proliferation inhibition of ASM cells. According to the analysis of the results of network pharmacology, quercetin in Polygonum cuspidatum has good oral bioavailability and drug-like properties, and most target proteins are enriched, which may also play a key role in the treatment of asthma (Table 1, Figure 2B). We found that quercetin at high dose (100uM) could 
A

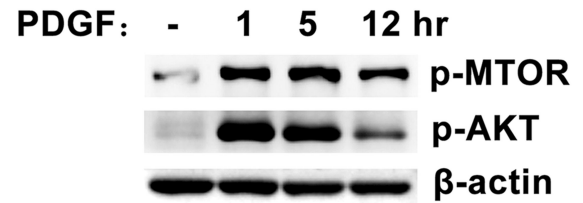

\section{C}

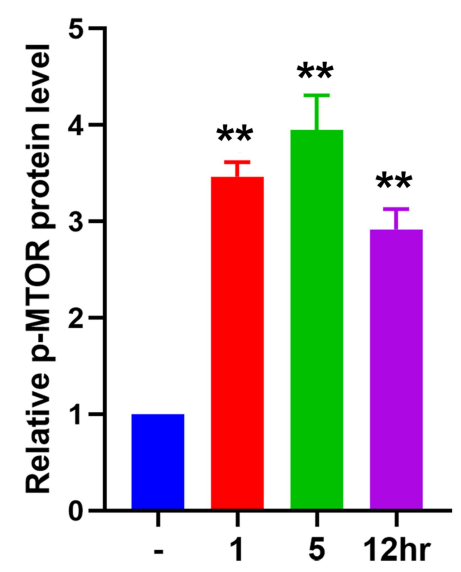

B

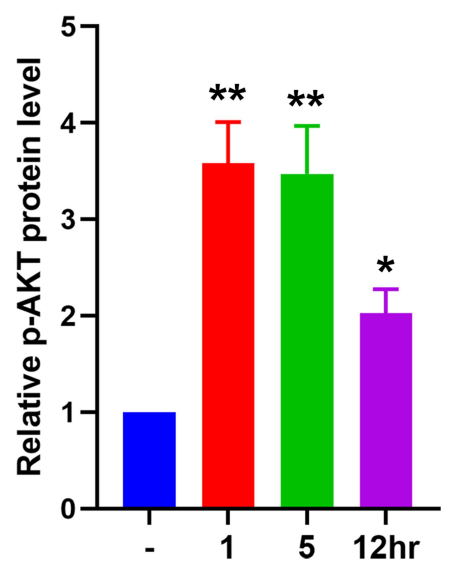

D

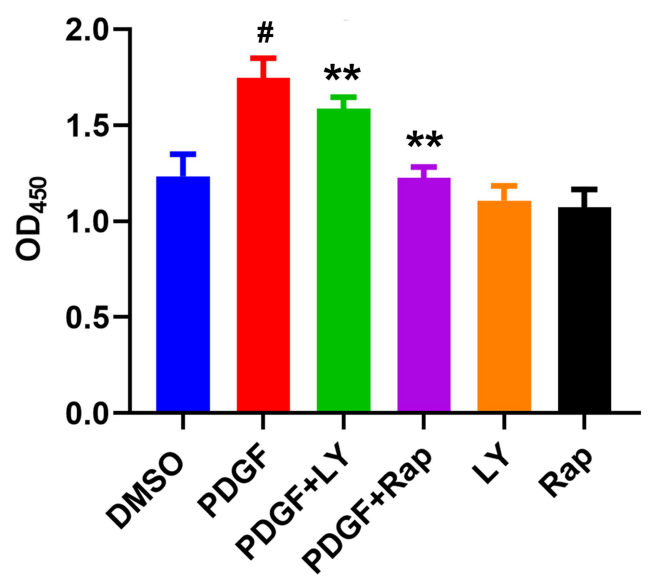

Figure 6 PDGF induced ASM cells proliferation by activating AKT/mTOR pathway. (A-C) ASM cells were treated with PDGF (I $\mathrm{ng} / \mathrm{mL}$ ) for Ih, 5h, and I $2 \mathrm{~h}$. The expression levels of $\mathrm{P}$-AKT and $\mathrm{p}-\mathrm{mTOR}$ were analyzed by Western blotting. The relative protein levels of P-AKT and P-mTOR were calculated by Image J, respectively and expressed as mean $\pm S D(n=3)$ of each group. $* P<0.05$ and $* * P<0.01$ vs control group. (D) ASM cells were treated with PDGF (IOng/mL) and LY294002 (I0 $\mu M)$, Rapamycin (500 $n M)$ for 5 h, respectively. Cell proliferation was detected by CCK-8. The data were expressed as mean \pm SD ( $n=8)$ of each group, ${ }^{* *} P<0.01$ vs $P D G F$ group, ${ }^{\#} P<0.0$ Ivs control group.

inhibit PDGF-induced ASM cell proliferation and AKT activation (Figure S1). Pan et al identified 7 ingredients identified from the crude extract of Polygonum cuspidatum including quercetin, polydatin, resveratrol, emodin, apigenin, rhein and catechin. ${ }^{25}$ We then verified the effects of other ingredients on the proliferation of ASM cells, and found that rhein and catechin could inhibit the proliferation of ASM cells whereas polydatin did not (Figure S2). Therefore, it is possible that the proliferation of ASM cells was inhibited by different active ingredients of Polygonum cuspidatum, which will need to be verified by further experiments.

According to the results of the network pharmacology screening, Polygonum cuspidatum may directly or indirectly affect asthma through 191 potential targets, such as AKT1, IL-6, VEGFA, MAPK3, CASP3, JUN, STAT3, and CASP8 (Figure 3). Polygonum cuspidatum is involved in the regulation of 165 KEGG signaling pathways, including the PI3K-AKT signaling pathway, apoptosis pathway and MAPK signaling pathway (Figure 4, Table 2), that play a therapeutic role in asthma. According to the enrichment analysis of the KEGG pathway, the PI3K-AKT signaling pathway was found to show the highest enrichment among the selected pathways with the highest correlation in the treatment of asthma by Polygonum cuspidatum, which showed the enrichment of 42 target proteins (Table S2). $\mathrm{AKT} /$ protein kinase $\mathrm{B}$ is a serine/threonine-specific protein kinase that plays an important role in regulating cell apoptosis and proliferation, and inhibition of the AKT 
A

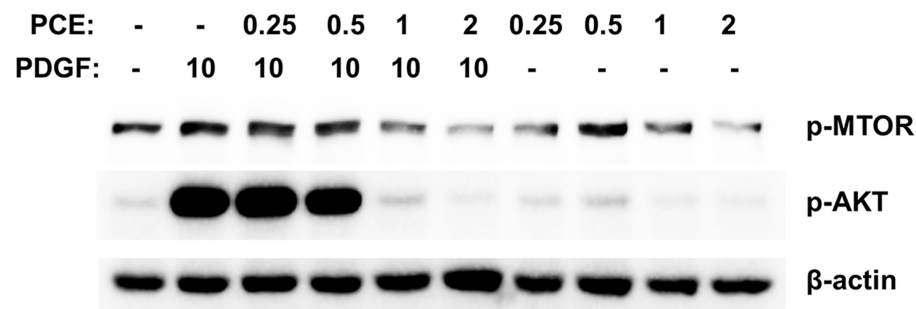

B

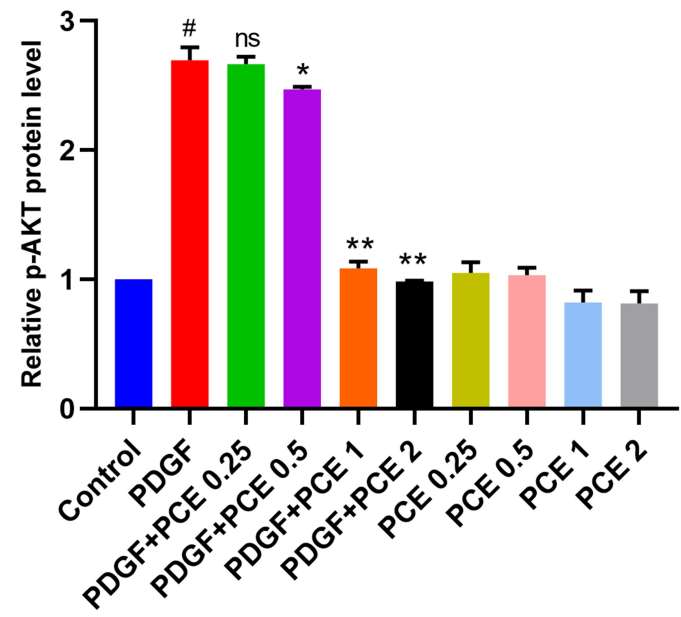

C

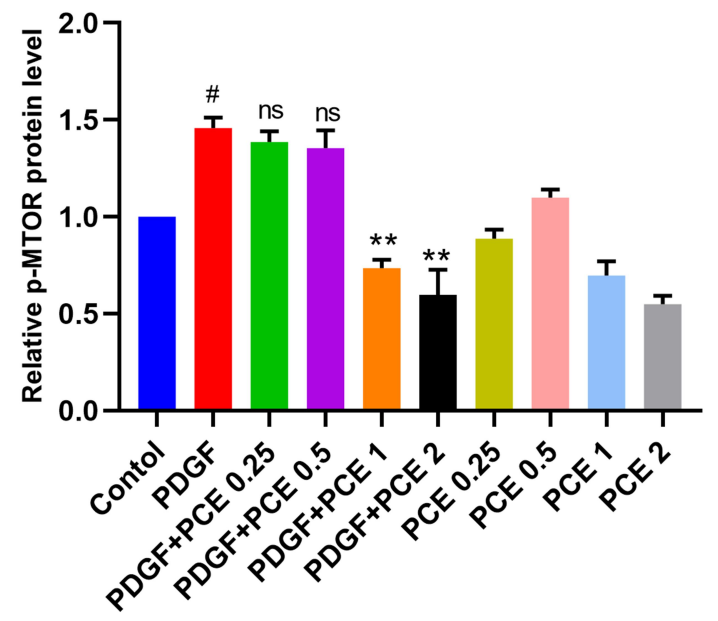

Figure 7 Polygonum cuspidatum extraction inhibited PDGF-induced AKT/mTOR activation. (A-C) ASM cells were treated with PDGF (IOng/mL) and Polygonum cuspidatum extraction $(0.25 \mathrm{mg} / \mathrm{mL}, 0.5 \mathrm{mg} / \mathrm{mL}, I \mathrm{mg} / \mathrm{mL}$ and $2 \mathrm{mg} / \mathrm{mL})$ for $5 \mathrm{~h}$, respectively. The expression levels of $\mathrm{p}-\mathrm{AKT}$ and $\mathrm{p}-\mathrm{mTOR}$ were analyzed by Western blotting. The relative protein levels of $p$-AKT and p-mTOR were calculated by Image $J$ and expressed as mean \pm SD $(n=3)$ of each group. ${ }^{\text {ns }} P>0.05$ vs PDGF group, ${ }^{*} P<0.05$ and $* * P<0.01$ vs PDGF group, ${ }^{\#} \mathrm{P}<0.0$ Ivs control group.

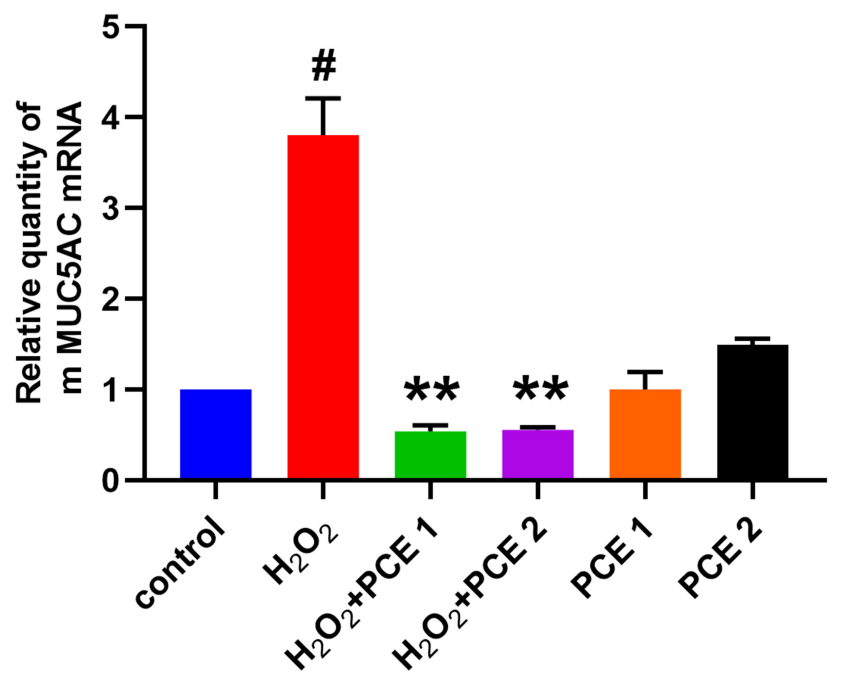

Figure 8 Polygonum cuspidatum extraction inhibited $\mathrm{H}_{2} \mathrm{O}_{2}$-induced MUC5AC expression. ASM cells were treated with $\mathrm{H}_{2} \mathrm{O}_{2}(80 \mu \mathrm{M})$ and Polygonum cuspidatum extraction ( $1 \mathrm{mg} / \mathrm{mL}$ and $2 \mathrm{mg} / \mathrm{mL}$ ) at different concentrations alone or in combination for $24 \mathrm{~h}$. The expression levels of MUC5AC were analyzed by Real-time PCR. The data were expressed as mean $\pm S D(n=3)$ of each group. ** $P<0.01$ vs $\mathrm{H}_{2} \mathrm{O}_{2}$ group, ${ }^{\#} \mathrm{P}<0.0$ Ivs control group. pathway is implicated in decreasing ASM cell proliferation in asthma. Ma L et al reported that ASM cells in bronchial sections from asthmatic patients show strong immunoreactivity for phospho-Ser473-AKT in immunohistochemical staining which indicated that AKT activation did occur in the airways of asthmatic patients. ${ }^{32}$ In our study, the analysis of AKT signaling demonstrated that the p-AKT level was significantly increased in PDGF-treated ASM cells, whereas treatment with an AKT inhibitor decreased PDGF-induced ASM cell proliferation. Our results supported the role of AKT in promoting ASM cell proliferation (Figure 6). We also observed that Polygonum cuspidatum and resveratrol inhibited the proliferation of rat airway ASM cells and significantly reversed PDGF-induced cell inhibition by inhibiting AKT. To further elucidate the pathway that mediated cell proliferation inhibition and apoptosis, we examined the downstream AKT pathway. Western 
A

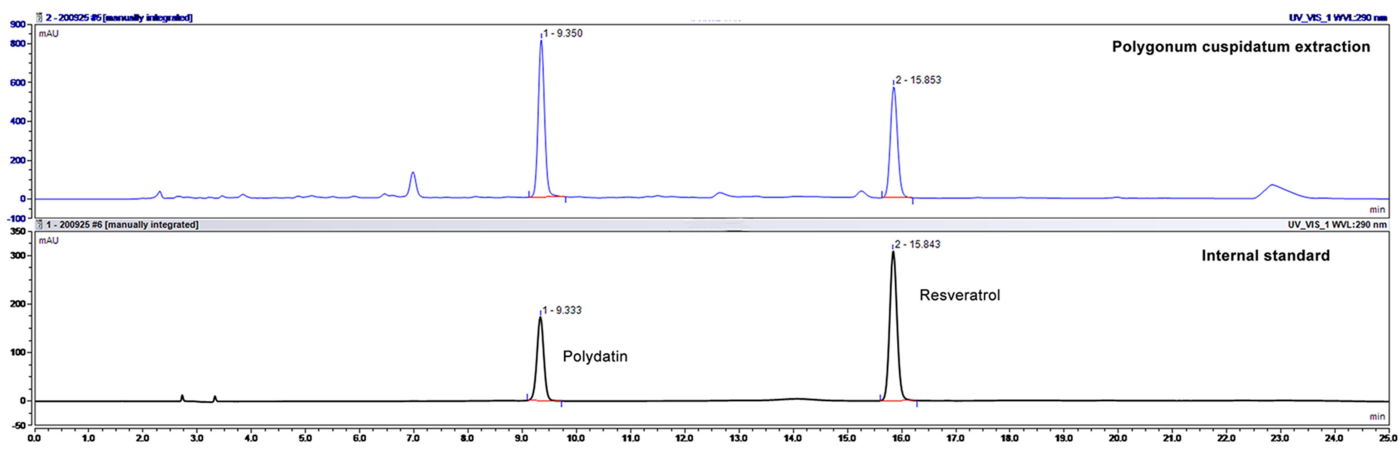

B

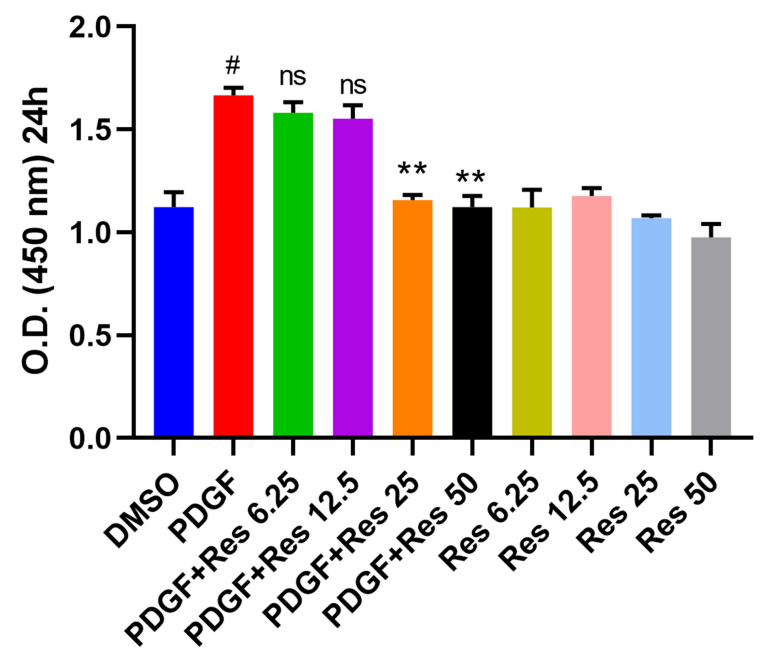

C

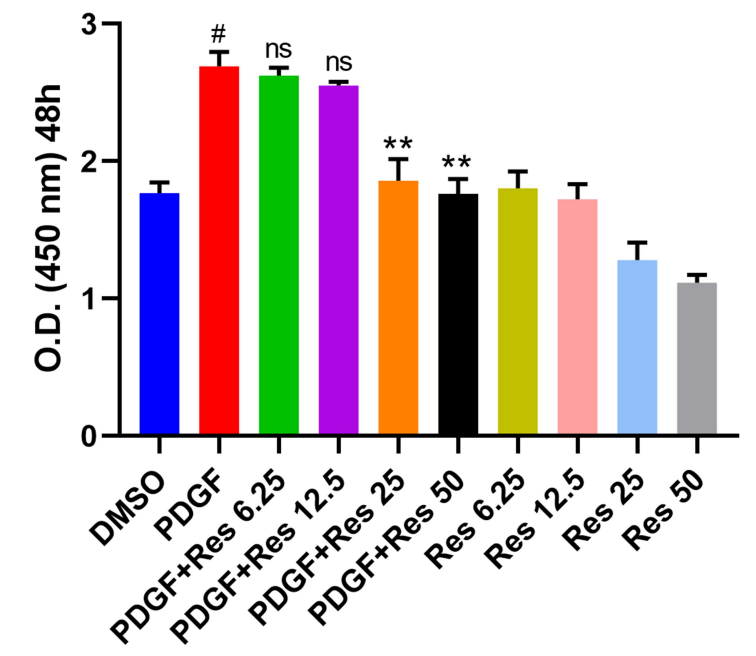

D

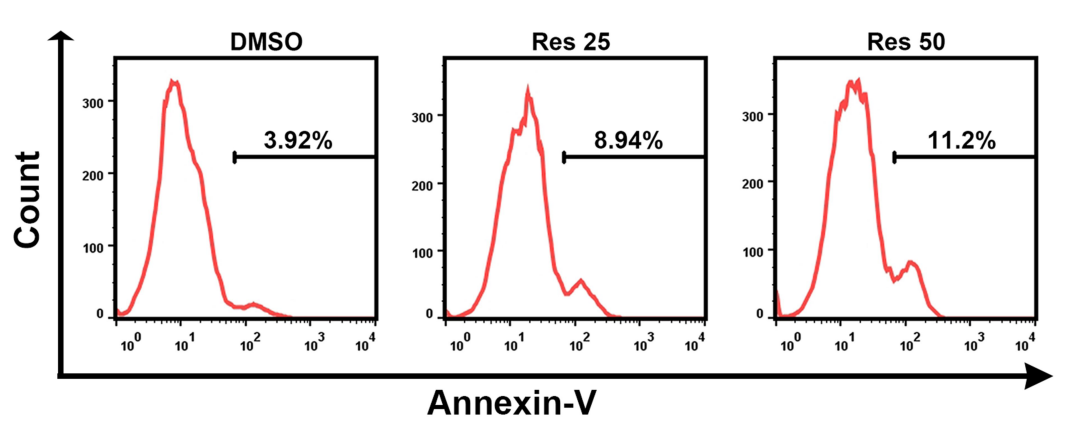

$\mathbf{E}$

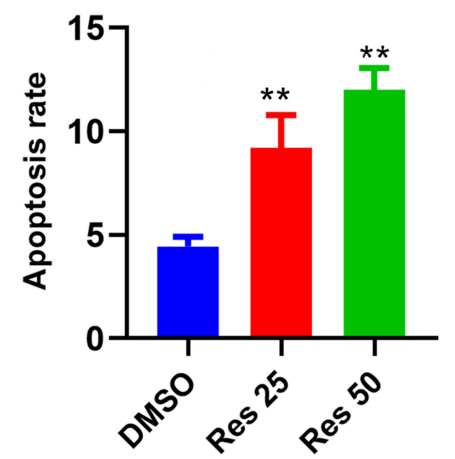

Figure 9 The effects of resveratrol on ASM cells proliferation and apoptosis. (A) HPLC chromatograms of resveratrol in Polygonum cuspidatum extraction. (B, C) ASM cells were treated with PDGF $(10 \mathrm{ng} / \mathrm{mL})$ and resveratrol $(6.25 \mu \mathrm{M}, 12.5 \mu \mathrm{M}, 25 \mu \mathrm{M}, 50 \mu \mathrm{M})$ at different concentrations alone or in combination for $24 \mathrm{~h}$ and $48 \mathrm{~h}$. Cell proliferation was detected by CCK-8. The data were expressed as mean $\pm \mathrm{SD}(\mathrm{n}=6)$ of each group, ${ }^{\mathrm{ns}} \mathrm{P}>0.05$ vs PDGF group, ${ }^{* *} \mathrm{P}<0.01$ vs PDGF group, ${ }^{\#} \mathrm{P}<0.0$ lvs $\mathrm{DMSO}$ group (control group). (D, E) The apoptosis of ASM cells were detected by Annexin-V staining after treatment with resveratrol $(25 \mu \mathrm{M}, 50 \mu \mathrm{M})$ for $48 \mathrm{~h}$. The data were expressed as mean $\pm S D(n=3)$ of each group, $* * P<0.01$ vs DMSO group (control group).

blotting analysis showed that the phosphorylation of p-mTOR was also reduced (Figure 7). These results indicate the potential signaling pathway involved in the treatment of asthma by Polygonum cuspidatum.
Mucin 5AC (MUC5AC) is one of the major gelatinforming mucins in airway mucus. ${ }^{33}$ Increased levels of MUC5AC protein had found to be present in the airways of asthma patients and contribute to the pathophysiology of 


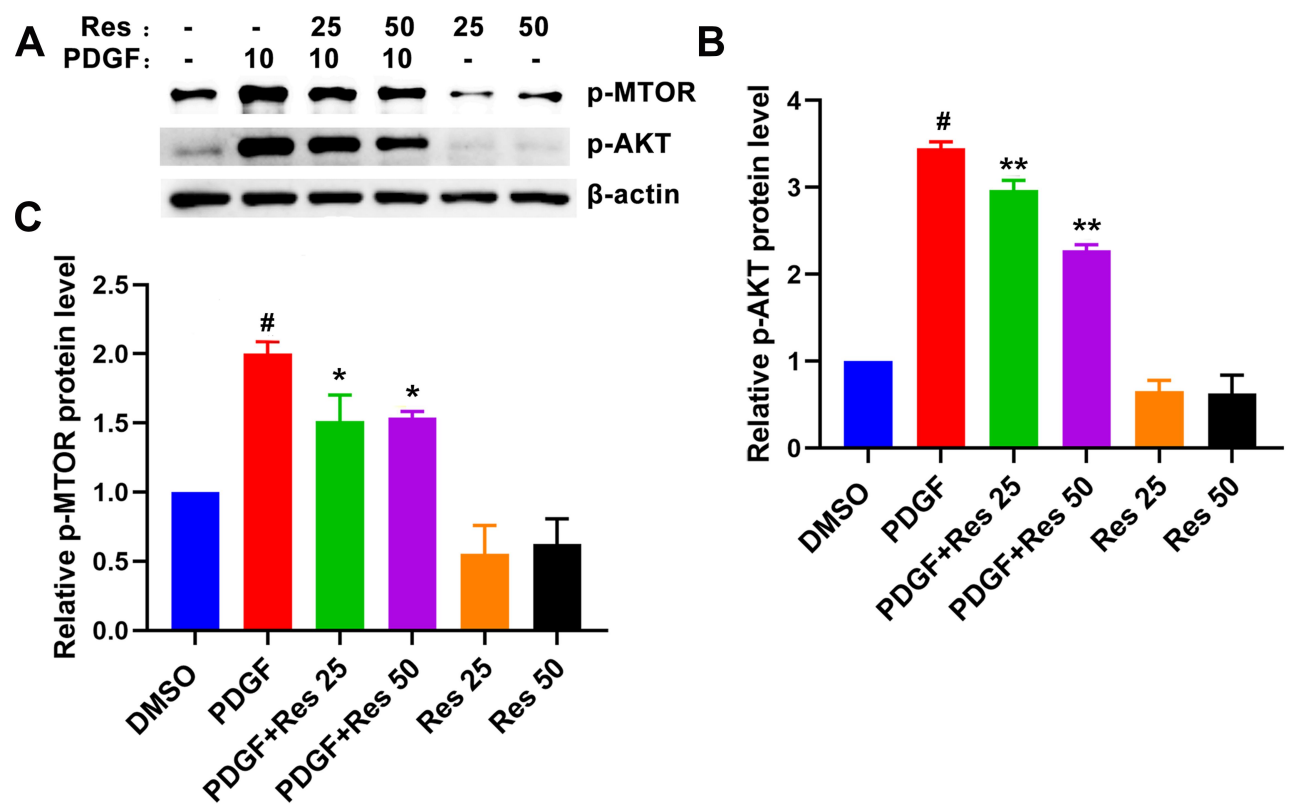

Figure 10 Resveratrol inhibited PDGF-induced AKT/mTOR activation. (A-C) ASM cells were treated with PDGF (I0 ng/mL) and Resveratrol (25 $\mu$ M, $50 \mu \mathrm{M})$ for $5 \mathrm{~h}$, respectively. The expression levels of $p$-AKT and p-mTOR were analyzed by Western blotting. The relative protein levels of $p$-AKT and $p$-mTOR were calculated by Image $\mathrm{J}$ and expressed as mean $\pm \mathrm{SD}(\mathrm{n}=3)$ of each group. ${ }^{*} \mathrm{P}<0.05$ and ${ }^{* *} \mathrm{P}<0.0 \mathrm{I}$ vs PDGF group, ${ }^{*} \mathrm{P}<0.0$ Ivs DMSO group (control group).

asthma such as airway mucus obstruction and reduction in lung function. ${ }^{33}$ Several studies reported that MAPK signaling pathway including JNK, ERK, and p38 was crucial signaling factors involved in MUC5AC expression. ${ }^{22,34}$ Mitogenactivated protein kinase (MAPK) is an important transmitter of extracellular signals from the cell surface to the nucleus, which could be activated by oxidative stress and a variety of pro-inflammatory factors. ${ }^{22,23,35}$ Through network pharmacology, we found that MAPK signaling pathway was involved in the treatment effect of Polygonum cuspidatum (Figure 4). Our group previously found that $\mathrm{H}_{2} \mathrm{O}_{2}$ can activate MAPK signaling pathway. ${ }^{13}$ Therefore, we activated MAPK through $\mathrm{H}_{2} \mathrm{O}_{2}$ and found that Polygonum cuspidatum could inhibit the expression of MUC5AC induced by $\mathrm{H}_{2} \mathrm{O}_{2}$. These results demonstrated the multiple anti-asthmatic roles of Polygonum cuspidatum. However, further investigation is still needed as there are some limitations in our study. The main limitation of our study is that we did not in vivo examine the anti-asthmatic role of Polygonum cuspidatum and its active ingredients in vivo. Based on network pharmacology analysis, Polygonum cuspidatum is likely to alleviate asthma through different mechanisms, such as anti-inflammatory activity, in addition to inhibiting ASM cell proliferation and MUC5AC expression. Therefore, further study should be performed to verify the role of Polygonum cuspidatum in asthmatic animal models.

\section{Conclusion}

Our results suggest that Polygonum cuspidatum could alleviate the symptoms of asthma such as ASM cells proliferation and MUC5AC expression through the mechanisms predicted by network pharmacology. These findings provide potential molecular and cellular evidence that Polygonum cuspidatum may be a promising anti-asthmatic drug to inhibit the proliferation of ASM and MUC5AC overexpression.

\section{Acknowledgments}

This work was supported by the Shanghai Science and Technology Committee (No.20ZR1450400, 1814 0904000, 17401970900), the Plan of Clinical Specialty Construction in Shanghai Putuo District Health System (No.2020tszk02), the Scientific Innovation Foundation of Putuo District (No.ptkwws201714), Shanghai Municipal Commission of Health and Family Planning (No.20174Y0239) and the Scientific Foundation of Putuo Hospital (No.2019301).

\section{Disclosure}

The authors declare that they have no conflicts of interest for this work. 


\section{References}

1. Murdoch JR, Lloyd CM. Chronic inflammation and asthma. Mutat Res. 2010;690(1-2):24-39. doi:10.1016/j.mrfmmm.2009.09.005

2. Fish JE, Peters SP. Airway remodeling and persistent airway obstruction in asthma. J Allergy Clin Immunol. 1999;104(3):509-516. doi:10.1016/S0091-6749(99)70315-5

3. Liu M, Liu G-Y, Hu S-Y, et al. [Treatment of Mild-to-moderate Bronchial Asthma (Heat Wheezing Syndrome) Patients at Acute Onset with Western Medicine Combined Danlong Oral Liquid: a Multi-center, Randomized Double- blinded, Positive-controlled Clinical Trial]. Zhongguo Zhong Xi Yi Jie He Za Zhi. 2015;35 (5):529-533.

4. Zhang HP, Wang L, Wang Z, et al. Chinese herbal medicine formula for acute asthma: a multi-center, randomized, double-blind, proof-ofconcept trial. Respir Med. 2018;140:42-49. doi:10.1016/j. rmed.2018.05.014

5. Wang L, Zheng X, Hui $\mathrm{Y}$, et al. Adjuvant treatment with Xiaoqinglong formula for bronchial asthma: protocol of systematic review and meta-analysis. Medicine. 2019;98(35):e17053. doi:10.1097/MD.0000000000017053

6. Asher BF, Seidman MD, Reddy WD, Omole FS. Integrative medical approaches to allergic rhinitis. Curr Opinion Otolaryngol Head Neck Surg. 2015;23(3):221-225. doi:10.1097/MOO.0000000000000152

7. Peng W, Qin R, Li X, Zhou H. Botany, phytochemistry, pharmacology, and potential application of Polygonum cuspidatum Sieb.et Zucc.: a review. $J$ Ethnopharmacol. 2013;148(3):729-745. doi:10.1016/j.jep.2013.05.007

8. Choi D-H, Han J-H, Yu K-H, et al. Antioxidant and Anti-Obesity Activities of Polygonum cuspidatum Extract through Alleviation of Lipid Accumulation on 3T3-L1 Adipocytes. J Microbiol Biotechn. 2020;30(1):21-30. doi:10.4014/jmb.1910.10040

9. Sheng Z, Ai B, Zheng L, et al. Capability of polygonum cuspidatum extract in inhibiting AGEs and preventing diabetes. Food Science \& Nutrition. 2019;7(6):2006-2016. doi:10.1002/fsn3.1029

10. Ma T-H, Sheng T, Tian C-M, et al. [Effect of ethanolic extract of Polygonum cuspidatum on acute gouty arthritis in mice through NLRP3/ASC/caspase-1 axis]. Zhongguo Zhong Yao Za Zhi. 2019;44 (3):546-552. doi:10.19540/j.cnki.cjemm.20180925.001

11. Liu B, Li S, Sui X, et al. Root Extract of Polygonum cuspidatum Siebold \& Zucc. Ameliorates DSS-Induced Ulcerative Colitis by Affecting NF-kappaB Signaling Pathway in a Mouse Model via Synergistic Effects of Polydatin, Resveratrol, and Emodin. Fron Pharmacol. 2018;9:347. doi:10.3389/fphar.2018.00347

12. Wang Y-L, Horng C-T, Hsieh M-T, et al. Autophagy and apoptotic machinery caused by Polygonum cuspidatum extract in cisplatin-resistant human oral cancer CAR cells. Oncol Rep. 2019;41 (4):2549-2557. doi:10.3892/or.2019.6985

13. Tang L, Zhu L, Zhang W, et al. Qi-Xian Decoction Upregulated E-cadherin Expression in Human Lung Epithelial Cells and Ovalbumin-Challenged Mice by Inhibiting Reactive Oxygen Species-Mediated Extracellular-Signal-Regulated Kinase (ERK) Activation. Med Sci Monit. 2020;26:e922003. doi:10.12659/ MSM.922003

14. Wang R, Lin J. Analysis of the Mechanism of Zhichuanling Oral Liquid in Treating Bronchial Asthma Based on Network Pharmacology. Evid Based Complement Alternat Med. 2020;2020:1875980. doi:10.1155/2020/1875980

15. Zeng $\mathrm{H}$, Wang $\mathrm{Y}, \mathrm{Gu} \mathrm{Y}$, et al. Polydatin attenuates reactive oxygen species-induced airway remodeling by promoting Nrf2-mediated antioxidant signaling in asthma mouse model. Life Sci. 2019;218:25-30. doi:10.1016/j.lfs.2018.08.013

16. Jiang H, Duan J, Xu K, Zhang W. Resveratrol protects against asthma-induced airway inflammation and remodeling by inhibiting the HMGB1/TLR4/NF-кB pathway. Exp Therapeutic med. 2019;18 (1):459-466. doi:10.3892/etm.2019.7594
17. Zhao X, Liu Z-P. Analysis of Topological Parameters of Complex Disease Genes Reveals the Importance of Location in a Biomolecular Network. Genes. 2019;10(2):2. doi:10.3390/genes10020143

18. Zhao L, Wu J, Zhang X, et al. The effect of Shenmai injection on the proliferation of Rat airway smooth muscle cells in asthma and underlying mechanism. BMC Complement Alternative Med. 2013;13 (1):221. doi:10.1186/1472-6882-13-221

19. Ni Z-H, Tang J-H, Chen G, et al. Resveratrol inhibits mucus overproduction and MUC5AC expression in a murine model of asthma. Mol Med Rep. 2016;13(1):287-294. doi:10.3892/ mmr.2015.4520

20. Lee HY, Kim IK, Yoon HK, et al. Inhibitory Effects of Resveratrol on Airway Remodeling by Transforming Growth Factor- $\beta /$ Smad Signaling Pathway in Chronic Asthma Model. Allergy Asthma Immunol Res. 2017;9(1):25-34. doi:10.4168/aair.2017.9.1.25

21. Yap HM, Lee YZ, Harith HH, et al. The geranyl acetophenone tHGA attenuates human bronchial smooth muscle proliferation via inhibition of AKT phosphorylation. Sci Rep. 2018;8(1):16640. doi:10.1038/s41598-018-34847-0

22. Shin I-S, Park J-W, Shin N-R, et al. Melatonin inhibits MUC5AC production via suppression of MAPK signaling in human airway epithelial cells. J Pineal Res. 2014;56(4):398-407. doi:10.1111/ jpi. 12127

23. Jang MK, Kim S-H, Lee K-Y, et al. The tyrosine phosphatase, SHP-1, is involved in bronchial mucin production during oxidative stress. Biochem Biophys Res Commun. 2010;393(1):137-143. doi:10.1016/j. bbrc.2010.01.102

24. Wu X, Li Q, Feng Y, Ji Q. Antitumor Research of the Active Ingredients from Traditional Chinese Medical Plant Polygonum Cuspidatum. Evid Based Complement Alter Med. 2018;2018:2313021. doi:10.1155/2018/2313021

25. Pan B, Shi X, Ding T, Liu L. Unraveling the action mechanism of polygonum cuspidatum by a network pharmacology approach. $\mathrm{Am}$ J Transl Res. 2019;11(11):6790-6811.

26. Lee M, Kim S, Kwon OK, et al. Anti-inflammatory and anti-asthmatic effects of resveratrol, a polyphenolic stilbene, in a mouse model of allergic asthma. Int Immunopharmacol. 2009;9 (4):418-424. doi:10.1016/j.intimp.2009.01.005

27. Alharris E, Alghetaa H, Seth R, et al. Resveratrol Attenuates Allergic Asthma and Associated Inflammation in the Lungs Through Regulation of miRNA-34a That Targets FoxP3 in Mice. Front Immunol. 2018;9:2992. doi:10.3389/fimmu.2018.02992

28. Tang L, Chen Q, Meng Z, et al. Suppression of Sirtuin-1 Increases IL-6 expression by activation of the akt pathway during allergic asthma. Cell Physiol Biochem. 2017;43(5):1950-1960. doi:10.1159/ 000484119

29. Aich J, Mabalirajan U, Ahmad T, et al. Resveratrol attenuates experimental allergic asthma in mice by restoring inositol polyphosphate 4 phosphatase (INPP4A). Int Immunopharmacol. 2012;14(4):438-443. doi:10.1016/j.intimp.2012.08.017

30. Ghanim H, Sia CL, Abuaysheh S, et al. An antiinflammatory and reactive oxygen species suppressive effects of an extract of Polygonum cuspidatum containing resveratrol. $J$ Clin Endocrinol Metab. 2010;95(9):E1-E8. doi:10.1210/jc.2010-0482

31. Bralley EE, Greenspan P, Hargrove JL, Wicker L, Hartle DK. Topical anti-inflammatory activity of Polygonum cuspidatum extract in the TPA model of mouse ear inflammation. J Inflamm. 2008;5:1. doi:10.1186/1476-9255-5-1

32. Ma L, Brown M, Kogut P, et al. Akt activation induces hypertrophy without contractile phenotypic maturation in airway smooth muscle. Am J Physiol Lung Cell Mol Physiol. 2011;300(5):L701-L709. doi:10.1152/ajplung.00119.2009

33. Welsh KG, Rousseau K, Fisher G, et al. MUC5AC and a glycosylated variant of MUC5B alter mucin composition in children with acute asthma. Chest. 2017;152(4):771-779. doi:10.1016/j.chest.201 7.07.001. 
34. Yang D, Jin M, Bai C, Zhou J, Shen Y. Peroxiredoxin 6 suppresses Muc5ac overproduction in LPS-induced airway inflammation through H2O2-EGFR-MAPK signaling pathway. Respir Physiol Neurobiol. 2017;236:84-90. doi:10.1016/j.resp.2016.11.012
35. Schindler JF, Monahan JB, Smith WG. p38 pathway kinases as anti-inflammatory drug targets. J Dent Res. 2007;86(9):800-811. doi: $10.1177 / 154405910708600902$

\section{Publish your work in this journal}

Drug Design, Development and Therapy is an international, peerreviewed open-access journal that spans the spectrum of drug design and development through to clinical applications. Clinical outcomes, patient safety, and programs for the development and effective, safe, and sustained use of medicines are a feature of the journal, which has also been accepted for indexing on PubMed Central. The manuscript management system is completely online and includes a very quick and fair peer-review system, which is all easy to use. Visit http://www. dovepress.com/testimonials.php to read real quotes from published authors.

Submit your manuscript here: https://www.dovepress.com/drug-design-development-and-therapy-journal 